\title{
Patient-reported outcomes from a randomized phase II study of the deferasirox film-coated tablet in patients with transfusion-dependent anemias
}

\author{
Ali T. Taher ${ }^{1,11^{*}}$, Raffaella Origa ${ }^{2}$, Silverio Perrotta ${ }^{3}$, Alexandra Kouraklis ${ }^{4}$, Giovan Battista Ruffo ${ }^{5}$, Antonis Kattamis ${ }^{6}$,
} Ai-Sim Goh? ${ }^{7}$ Vicky Huang ${ }^{8}$, Aiesha Zia ${ }^{9}$, Raquel Merino Herranz ${ }^{9}$ and John B. Porter ${ }^{10}$

\begin{abstract}
Background: Adherence to long-term chelation therapy in transfusion-dependent patients is critical to prevent iron overload-related complications. Once-daily deferasirox dispersible tablets (DT) have proven long-term efficacy and safety in patients $\geq 2$ years old with chronic transfusional iron overload. However, barriers to optimal adherence remain, including palatability, preparation time, and requirements for fasting state. A new film-coated tablet (FCT) formulation was developed, swallowed once daily (whole/crushed) with/without a light meal.

Methods: The open-label, Phase II ECLIPSE study evaluated patient-reported outcomes (PROs) in transfusiondependent thalassemia or lower-risk myelodysplastic syndromes patients randomized 1:1 to receive deferasirox DT or FCT over 24 weeks as a secondary outcome of the study. Three PRO questionnaires were developed to evaluate both deferasirox formulations: 1) Modified Satisfaction with Iron Chelation Therapy Questionnaire; 2) Palatability Questionnaire; 3) Gastrointestinal (Gl) Symptom Diary.

Results: One hundred seventy three patients were enrolled; 87 received the FCT and 86 the DT formulation. FCT recipients consistently reported better adherence (easier to take medication, less bothered by time to prepare medication and waiting time before eating), greater satisfaction/preference (general satisfaction and with administration of medicine), and fewer concerns (less worry about not swallowing enough medication, fewer limitations in daily activities, less concern about side effects). FCT recipients reported no taste or aftertaste and could swallow all their medicine with an acceptable amount of liquid. Gl summary scores were low for both formulations.

Conclusions: These findings suggest a preference in favor of the deferasirox FCT formulation regardless of underlying disease or age group. Better patient satisfaction and adherence to chelation therapy may reduce iron overloadrelated complications.
\end{abstract}

Trial registration: ClinicalTrials.gov identifier: NCT02125877; registered April 26, 2014.

Keywords: Iron chelation, Iron overload, Deferasirox, Patient-reported outcomes

\footnotetext{
* Correspondence: ataher@aub.edu.lb

'Department of Internal Medicine, Director - Fellowship and Residents

Research Program, Faculty of Medicine American University of Beirut Medical

Center, Beirut, Lebanon

${ }^{11}$ Emory School of Medicine, Atlanta, USA

Full list of author information is available at the end of the article
}

(c) The Author(s). 2018 Open Access This article is distributed under the terms of the Creative Commons Attribution 4.0 International License (http://creativecommons.org/licenses/by/4.0/) which permits unrestricted use, distribution, and reproduction in any medium, provided you give appropriate credit to the original author(s) and the source, provide a link to the Creative Commons license, and indicate if changes were made. The Creative Commons Public Domain Dedication waiver (http://creativecommons.org/publicdomain/zero/1.0/) applies to the data made available in this article, unless otherwise stated. 


\section{Background}

In patients with hematological disorders requiring red blood cell transfusions to treat anemia, such as those with transfusion-dependent thalassemia (TDT) and myelodysplastic syndromes (MDS), long-term iron chelation therapy is necessary to remove excess iron and prevent organ failure [1]. Because iron overload is a chronic condition and the benefits of chelation therapy are not immediately perceivable, compliance with chelation therapy has a positive influence on the occurrence of iron overload-related complications and survival [2-4]. As such, a convenient and well-tolerated chelation regimen is required for optimal patient outcomes.

The once-daily oral deferasirox dispersible tablet (DT) formulation (Exjade ${ }^{\odot}$ ) has been used in clinical practice for over a decade, with a well-established efficacy and safety profile in patients $\geq 2$ years of age with chronic transfusional iron overload [5-13]. Oral deferasirox DT offered an improvement over parenteral deferoxamine (DFO, Desferal ${ }^{\oplus}$ ), particularly in terms of compliance, convenience, patient satisfaction, and health-related quality of life [2, 14-16]. However, barriers still exist to optimal adherence with deferasirox DT, including the need to take the drug in a fasting state, requirements for careful dispersion prior to ingestion, the chalky consistency, and suboptimal gastrointestinal (GI) tolerability [17]. In recognition of the importance of patient adherence to chelation therapy, a film-coated tablet (FCT) formulation for oral administration (either whole or crushed and mixed with soft foods) was developed as an alternative treatment option intended to improve patient acceptability and therefore compliance. Both FCT and DT contain the same active substance (deferasirox) and are administered once daily. Dose is adjusted according to body weight and response to treatment [1820]. In contrast to the DT, the FCT does not contain the excipients lactose and sodium lauryl sulphate and can be taken with or without a light meal [19, 20] offering a more simple and convenient mode of administration with potential improvements in GI tolerability.

The Phase II, randomized open-label ECLIPSE study demonstrated similar safety profiles for the FCT and DT, including acceptable GI tolerability for both formulations [20]. The study provided evidence that patients receiving the FCT remained on treatment for longer and were more compliant than those receiving the DT. The patientreported outcome (PRO) analyses also indicated a benefit in favor of the deferasirox FCT, including greater adherence, greater satisfaction, fewer concerns and better palatability [20]. Furthermore, a post-marketing assessment of safety outcomes from data available in the USA (where the FCT became available in April 2015), demonstrated a tendency towards better tolerability with the FCT, particularly in the GI tract [21].
Here, we report analyses from the individual response categories of the various PRO instruments used for the ECLIPSE study, specifically the modified Satisfaction with Iron Chelation Therapy (mSICT) and Palatability Questionnaire items, as well as the GI Symptom Diary. These analyses were performed to better understand the drivers of the improved PROs observed in patients receiving the FCT versus those receiving DT.

\section{Methods \\ Patients}

Key inclusion and exclusion criteria have been described elsewhere [20]. In summary, iron overloaded and previously chelated or chelation-naïve male and female patients (aged $\geq 10$ years) with TDT or revised International Prognostic Scoring System very-low, low- or intermediate-risk MDS were enrolled. Key exclusion criteria were: creatinine clearance below contraindication limit as per local label (<60 $\mathrm{mL} / \mathrm{min}$ or $<40 \mathrm{~mL} / \mathrm{min}$ ); serum creatinine $>1.5 \times$ upper limit of normal (ULN); alanine aminotransferase $>5 \times$ ULN (unless liver iron concentration confirmed as $>10 \mathrm{mg} \mathrm{Fe} / \mathrm{g}$ dry weight $\leq 6$ months prior to screening); urine protein/urine creatinine ratio $>0.5 \mathrm{mg} / \mathrm{mg}$; or impaired GI function.

\section{Study design}

ECLIPSE was a 24-week, open-label, randomized, multicenter, two-arm, Phase II study that evaluated the deferasirox FCT and DT formulations as previously described [20]. Randomization was stratified by underlying disease and prior chelation treatment. As per protocol, deferasirox DT (taken on an empty stomach, at least $30 \mathrm{~min}$ before the next meal) was to be initiated at $20 \mathrm{mg} / \mathrm{kg} /$ day in chelation-naïve patients; pretreated patients were to be initiated at a dose equivalent to their prior chelation treatment (taking conversion rules into account, e.g., $20 \mathrm{mg} / \mathrm{kg} /$ day deferasirox equivalent to $\sim 40 \mathrm{mg} / \mathrm{kg} /$ day DFO) following a 5-day washout period. As per protocol, deferasirox FCT (taken with or after a light meal) was to be initiated at $14 \mathrm{mg} / \mathrm{kg} /$ day in chelation-naïve patients; pre-treated patients were to be initiated at a dose equivalent to their prior chelation treatment (taking conversion rules into account, e.g., $20 \mathrm{mg} / \mathrm{kg} /$ day DT equivalent to $14 \mathrm{mg} / \mathrm{kg} /$ day FCT; conversion factor 1.43) following a 5 -day washout period. Dose adjustments to improve treatment response based on serum ferritin levels and the investigator's judgement were recommended every 4 weeks for chelation-naïve patients, and every 3 months for pre-treated patients, in increments of 5$10 \mathrm{mg} / \mathrm{kg} /$ day for DT or $3.5-7 \mathrm{mg} / \mathrm{kg} /$ day for FCT, up to a maximum dose of $40 \mathrm{mg} / \mathrm{kg} /$ day for DT and $28 \mathrm{mg} / \mathrm{kg} /$ day for FCT. 
The study was conducted in accordance with Good Clinical Practice guidelines and the Declaration of Helsinki and was approved by independent ethics committees at participating sites. Patients (or parents/guardians) provided written, informed consent prior to enrollment.

\section{PRO instruments}

Evaluation of both deferasirox formulations on patient satisfaction, palatability, and GI symptoms was conducted as a secondary endpoint of the study using PRO questionnaires. Three PRO questionnaires were developed: 1) mSICT; 2) Palatability Questionnaire; and 3) GI Symptoms Diary. Patients completed all PRO questions via a hand-held electronic device either at home (daily diaries) or during scheduled site visits. The instruments underwent full qualitative evaluation [22]; linguistic and psychometric evaluation of the PRO questionnaires was conducted during the trial, supporting the reliability and construct-related validity of the scores [23].

The mSICT questionnaire used five-point response scales to assess adherence (six questions), satisfaction/ preference (two questions), and concern domains (three questions). Domain scores were calculated by summing the scores from all questions within the domain; higher scores in adherence (range 6-30) and satisfaction/preference (range 2-10) domains indicated worse outcomes, higher scores in concern domain (range 3-15) indicated fewer concerns. The Palatability Questionnaire consisted of four items: taste and aftertaste of the medication (5-point response scale: $1=$ very good to $5=$ very bad), whether the medication was taken (i.e., whether the patient vomited after swallowing medication or not) and how the patient perceived the amount of medication to be taken (not enough, just enough, or too much). Overall palatability score ranged from 0 (worst) to 11 (best). These two questionnaires were completed at the start of treatment [SOT], which was week 2 after the first dose (or week 3 if missing), week 3, 13, and end of treatment (within 7 days of the last dose).

The GI Symptom Diary, completed daily, consisted of six items; five items (pain in your belly, nausea, vomiting, constipation, diarrhea) rated on an 11-point scale (0 = best, $10=$ worst) and the sixth item, bowel movement frequency during the past $24 \mathrm{~h}$, using seven response options $0=0$ (none), $1=1,2=2,3=3,4=4,5=5-10$ and $6=\geq 11$. The overall GI summary score was calculated from the responses to the five items that used the 11-point scale, ranging from 0 to 50 , with higher scores indicating worse symptoms. Item six was analysed as a standalone item.

Summary scores for each of the three PRO questionnaires were also summarized by underlying anemia (thalassemia or MDS), and age categories (10 to $<13,13$ to $<18,18$ to $<50,50$ to $<65$ and $\geq 65$ years).

\section{Statistical evaluations}

Standard descriptive analyses were performed. No hypothesis was tested. The minimally important difference (MID) was calculated (utilizing $1 / 2$ standard deviation [SD] [24] and standard error of measurement [25]) for overall scores for the mSICT and palatability summary score, representing the smallest difference between the two treatment groups that is considered clinically important or that implies treatment benefit [26]. MID scores $>1$ point for the mSICT domains and palatability scores and MID scores of 0.24 to 0.39 for the GI symptom score indicate a meaningful difference between groups. The full analysis set (FAS) includes all patients randomized to receive treatment, $\mathrm{N}$. The term 'evaluable patients' refers to those patients who provided a response to the PRO instruments, as indicated by the investigator. A supportive analysis was conducted to assess the robustness of the PRO results. Missing PRO scores (from mSICT, palatability and GI PRO questionnaire) were imputed using a multiple imputation method under missing at random assumption (MAR, i.e. the probability of missing data is unrelated to the values of PRO score). Given that the missing data patterns observed in the three PRO measures were monotone and intermittent (Table 1), an assessment based on the reasons for missing PRO measures was done, which supported MAR as an acceptable assumption to assess the robustness of the main analysis of PRO measures collected during the trial.

\section{Results}

\section{Patient disposition and PRO instrument response}

In total, 173 patients from 16 countries worldwide were randomized 1:1 to receive deferasirox DT $(N=86)$ or FCT $(N=87)$ treatment. Overall, $73(84.9 \%)$ and 77 (88.5\%) patients in the DT and FCT arm, respectively, completed the treatment period. The treatment groups were balanced with respect to type of anemia and prior chelation therapy, with 70 TDT patients in each arm and 77 and 79 previously chelated patients in the DT and FCT arm, respectively.

A similar proportion of patients completed the three questionnaires for both formulations at the SOT and at the end of treatment (EOT), with the response rate generally lower at the end of the study (Table 1). PRO results obtained using imputed scores, to account for missing PROs, were similar to the main PRO analyses (Fig. 1).

mSICT and Palatability Questionnaire mean domain results Completion of these two PRO questionnaires declined from $\sim 80 \%$ at SOT to $70 \%$ at EOT in both treatment arms (Table 1). As previously reported, patients receiving the FCT consistently reported greater adherence, 
Table 1 Completion of the three PRO instruments at each assessment during the study

\begin{tabular}{|c|c|c|c|c|c|c|}
\hline \multirow[t]{2}{*}{ Week } & \multicolumn{2}{|l|}{$\mathrm{mSICT}$} & \multicolumn{2}{|l|}{ Palatability } & \multicolumn{2}{|c|}{ Gl Symptom Diary* } \\
\hline & $\begin{array}{l}\text { Deferasirox DT } \\
N=86 \\
\mathrm{n}(\%)\end{array}$ & $\begin{array}{l}\text { Deferasirox FCT } \\
N=87 \\
\mathrm{n}(\%)\end{array}$ & $\begin{array}{l}\text { Deferasirox DT } \\
N=86 \\
\mathrm{n}(\%)\end{array}$ & $\begin{array}{l}\text { Deferasirox FCT } \\
N=87 \\
\mathrm{n}(\%)\end{array}$ & $\begin{array}{l}\text { Deferasirox DT } \\
N=86 \\
\mathrm{n}(\%)\end{array}$ & $\begin{array}{l}\text { Deferasirox FCT } \\
N=87 \\
\mathrm{n}(\%)\end{array}$ \\
\hline-2 & & & & & $60(69.8)$ & $59(67.8)$ \\
\hline-1 & & & & & $62(72.1)$ & $52(59.8)$ \\
\hline 1 & & & & & $70(81.4)$ & $69(79.3)$ \\
\hline 2 & 70 (81.4) & $70(80.5)$ & $69(80.2)$ & $70(80.5)$ & $65(75.6)$ & 71 (81.6) \\
\hline 3 & $58(67.4)$ & $51(58.6)$ & $57(66.3)$ & $51(58.6)$ & $64(74.4)$ & $66(75.9)$ \\
\hline 4 & & & & & $60(69.8)$ & $64(73.6)$ \\
\hline 5 & & & & & $58(67.4)$ & 64 (73.6) \\
\hline 6 & & & & & $64(74.4)$ & $57(65.5)$ \\
\hline 7 & & & & & $57(66.3)$ & $53(60.9)$ \\
\hline 8 & & & & & 59 (68.6) & $51(58.6)$ \\
\hline 9 & & & & & $55(64.0)$ & $49(56.3)$ \\
\hline 10 & & & & & $53(61.6)$ & $49(56.3)$ \\
\hline 11 & & & & & $51(59.3)$ & $44(50.6)$ \\
\hline 12 & & & & & $51(59.3)$ & $45(51.7)$ \\
\hline 13 & $59(68.6)$ & $64(73.6)$ & 59 (68.6) & $62(71.3)$ & $49(57.0)$ & $50(57.5)$ \\
\hline 14 & & & & & $51(59.3)$ & $44(50.6)$ \\
\hline 15 & & & & & $49(57.0)$ & $43(49.4)$ \\
\hline 16 & & & & & $48(55.8)$ & $41(47.1)$ \\
\hline 17 & & & & & $44(51.2)$ & $40(46.0)$ \\
\hline 18 & & & & & $44(51.2)$ & $38(43.7)$ \\
\hline 19 & & & & & $40(46.5)$ & $37(42.5)$ \\
\hline 20 & & & & & $40(46.5)$ & $39(44.8)$ \\
\hline 21 & & & & & $39(45.3)$ & $37(42.5)$ \\
\hline 22 & & & & & $38(44.2)$ & $35(40.2)$ \\
\hline 23 & & & & & $36(41.9)$ & $34(39.1)$ \\
\hline 24 & $63(73.3)$ & $60(69.0)$ & $63(73.3)$ & $60(69.0)$ & $32(37.2)$ & $26(29.9)$ \\
\hline
\end{tabular}

SOT for the mSICT and palatability were defined as the first PRO assessment at week 2 (or week 3 if missing); for the GI Symptom Diary, week 1 was taken as SOT; "includes patients with at least four complete daily responses. DT Dispersible tablet, FCT Film-coated tablet, GI Gastrointestinal, PRO Patient-reported outcome, mSICT modified Satisfaction with iron chelation therapy, SOT Start of treatment

satisfaction/preference and fewer concerns than patients receiving the DT. The difference in scores between treatment groups was $>1$ point $(\mathrm{MID}=1)$ at almost all visits for all three domains, indicating a clinically meaningful difference (Fig. 1a, b and c) [20]. Mean palatability summary scores in the FCT treatment arm were consistently high $(\sim 11)$ compared with the DT arm $(\sim 8-9)$ (Fig. 1d) [20]. The difference in scores between treatment groups was $>1$ point $(\mathrm{MID}=1)$, indicating a clinically meaningful difference. In all mean domain scores for the two questionnaires, the $95 \%$ confidence intervals did not overlap at most time points analysed. Analysis of summary scores by underlying anemia or age group did not reveal any notable differences compared to the overall findings (see Additional file 1: Table S2).
Item results from the $\mathrm{mSICT}$ adherence domain

To better understand the improved $\mathrm{mSICT}$ and Palatability Questionnaire mean domain results with FCT versus DT, patient responses to individual questionnaire items were evaluated. The adherence domain consisted of six questions related to: trouble remembering to take the medication, thinking about stopping the medication, following their doctor's instructions (including reasons for not always taking the medication, if a patient indicated they did not 'always' take their medication as instructed), how hard/easy it was to take the medication, how bothered they were about the time to prepare the medication and the time to wait to eat food. Overall, patients in the FCT arm reported consistent mean adherence scores from SOT (7.6) to EOT (7.5), whereas 

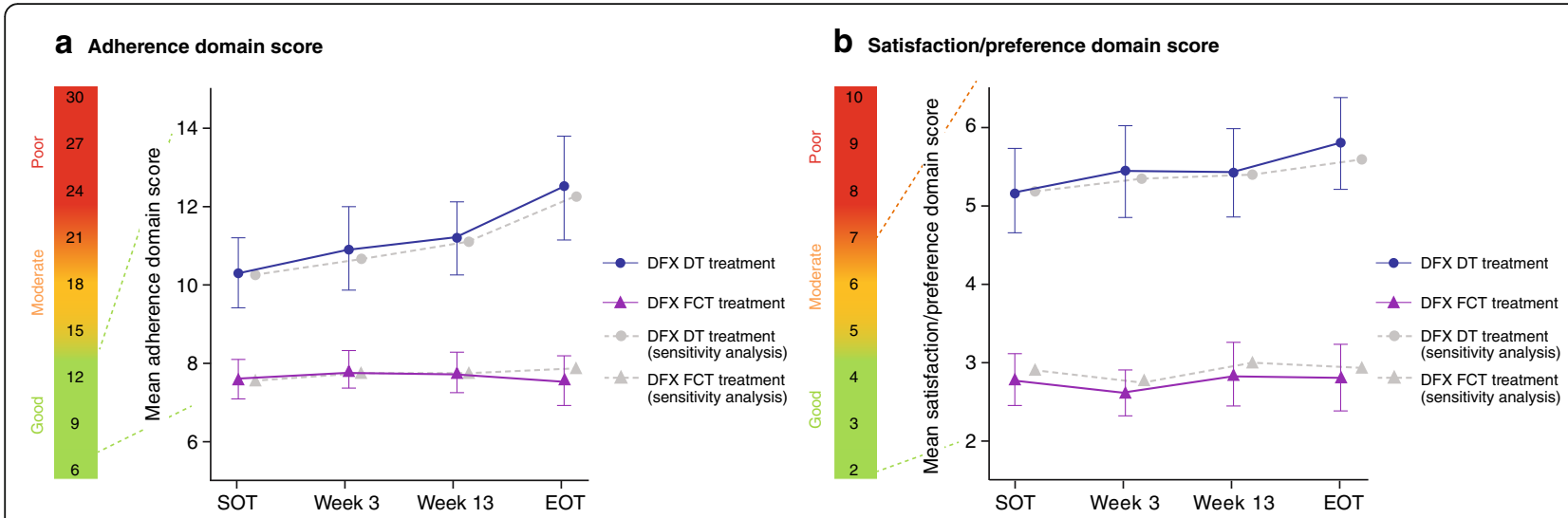

C Concerns domain score

d Palatability summary score
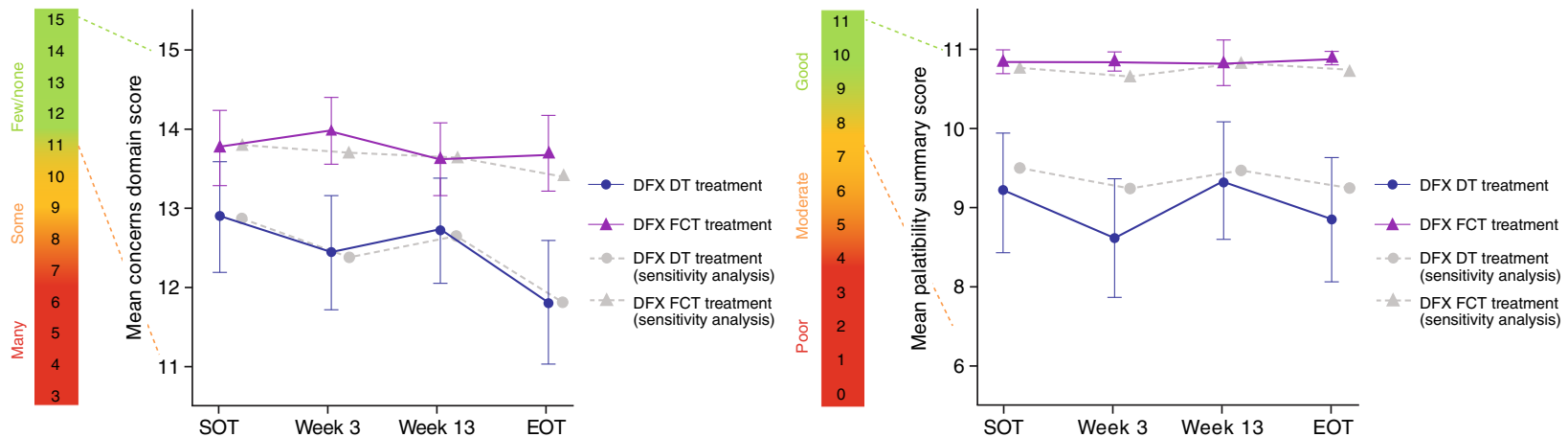

e GI symptom score
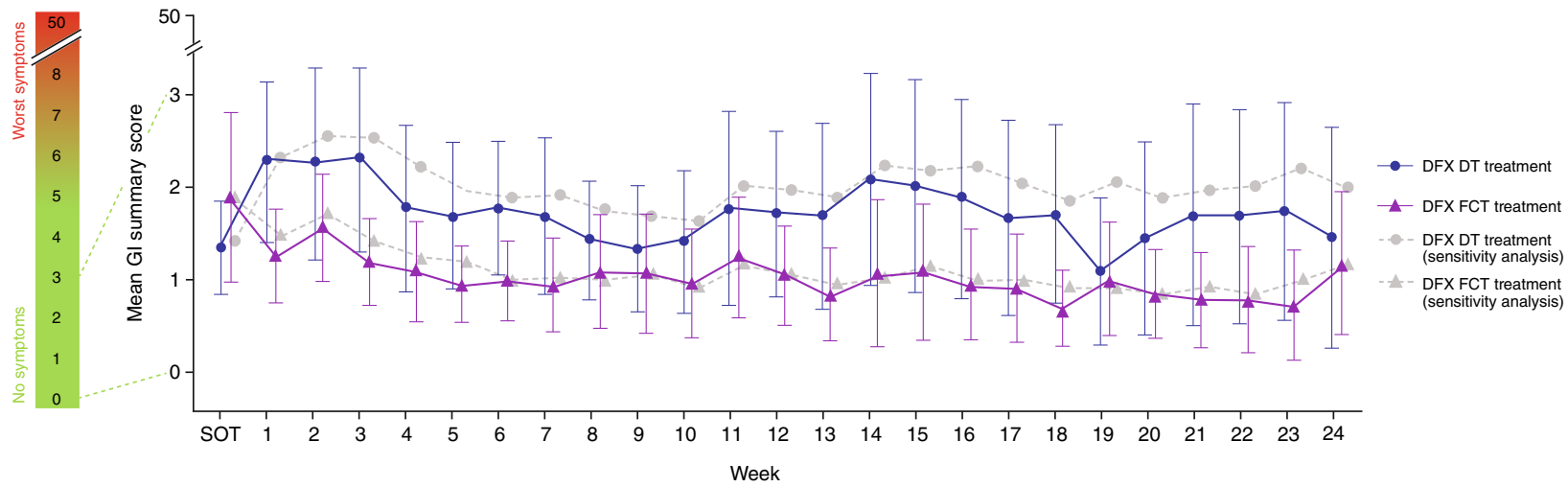

Fig. 1 Mean $\pm 95 \% \mathrm{Cl}$ domain scores for patient-reported outcomes (adherence, satisfaction/preference, and concern) (a-c), mean palatability score (d), and mean gastrointestinal symptom scores (e). For adherence (a; scale 6-30), satisfaction/preference (c; scale 2-10), and Gl symptoms (e; scale 0-50), higher scores indicate worse outcomes/symptoms. For concern (c; scale 3-15) and palatability (d; scale 0-11), higher scores indicate fewer concerns and better palatability. a-d, SOT was defined as week 2 assessment. If missing, then the week 3 assessment was considered SOT. e, SOT was defined as week 1 score. If missing, then the week 2 score was considered SOT. Cl, confidence interval; DFX, deferasirox; DT, dispersible tablet; EOT, end of treatment; FCT, film-coated tablet; SOT, start of treatment. Figure adapted from Taher AT et al. Am J Hematology 2017;92(5):420-428. Published with kind permission of John Wiley \& Sons @2017 The Authors, American Journal of Hematology, Published by Wiley Periodicals, Inc.

overall scores in the DT arm worsened by EOT (12.5) compared to SOT (10.3; Fig. 1a) and were slightly higher (indicating worse outcomes).

Most patients in both treatment arms reported 'never thinking about stopping medication' at SOT. By EOT, the proportion of DT recipients who reported 'never thinking about stopping medication' was $44 \cdot 2 \%(n=38 / 86[n=38 /$ $63,60 \cdot 3 \%$ evaluable patients]) compared with $60.9 \%(n=$ $53 / 87$ [ $n=53 / 60,88.3 \%$ evaluable patients]) of FCT recipients (Fig. 2a). The majority of FCT recipients $(n=65 / 87$, 
$74.7 \%$ at SOT $[\mathrm{n}=65 / 70,92.9 \%$ evaluable patients]; $n=$ $55 / 87,63.2 \%$ at EOT $[\mathrm{n}=55 / 60,91.7 \%$ evaluable patients $]$ ) found it 'very easy or easy' to take their medication, in comparison to DT recipients $(n=45 / 86,52.3 \%$ at SOT $[n=45 / 70,64.3 \%$ evaluable patients]; $n=29 / 86$, $33.7 \%$ at EOT $[n=29 / 63,46.0 \%$ evaluable patients]; Fig. 2b). More patients in the FCT group were 'not bothered at all' about the time it took to prepare medication (Fig. 2c) or by the time they had to wait to eat (Fig. 2d) than patients in the DT group at both SOT and EOT.

A similar proportion of patients in both treatment arms reported to 'always' follow their doctor's instructions (Fig. 2e), both at SOT (FCT: $n=56 / 87,64.4 \%$ [n $=56 / 70,80.0 \%$ evaluable patients] and DT: $n=58 / 86$, $67.4 \%[\mathrm{n}=58 / 70,82.9 \%$ evaluable patients] $)$ and EOT (FCT: $n=50 / 87,57.5 \% \quad[n=50 / 60,83.3 \%$ evaluable patients] and DT: $n=48 / 86,55 \cdot 8 \%[n=48 / 63,76.2 \%$ evaluable patients]). In both treatment arms, the proportion of patients 'rarely or never' having trouble remembering to take medication was similar (Fig. 2f) at SOT (FCT: $n=61 / 87,70 \cdot 1 \%$ [n $=61 / 70,87 \cdot 1 \%$ evaluable patients $]$ and DT: $n=61 / 86,70.9 \%[n=61 / 70,87.1 \%$ evaluable patients]), declining by EOT in the DT arm (FCT: $n=53 / 87,60.9 \% \quad[\mathrm{n}=53 / 60,88.3 \%$ evaluable patients $]$ and DT: $n=46 / 86,53.5 \%[n=46 / 63,73.0 \%$ evaluable patients]).

\section{Item results from the $\mathrm{mSICT}$ satisfaction domain}

The satisfaction domain consisted of three questions related to: satisfaction with how they took the medication, how satisfied/dissatisfied they were with the medication in general, and which type of medication did they like the best (see Overall Preference). Overall, patients in the FCT arm reported consistent mean satisfaction scores from SOT (2.8) to EOT (2.9), whereas overall scores in the DT arm worsened at EOT (5.8) compared to $\operatorname{SOT}(5 \cdot 2$, Fig. 1b) and were generally higher (indicating worse outcome).

More patients in the FCT than the DT group were 'very satisfied' or 'satisfied' with taking their medication at SOT (FCT: $n=67 / 87,77.0 \%[n=67 / 70,95.7 \%$ evaluable patients $]$ and DT: $n=32 / 86,37.2 \%[n=32 / 70$, $45.7 \%$ evaluable patients]) and EOT (FCT: $n=54 / 87$, $62.1 \%[n=54 / 60,90.0 \%$ evaluable patients] and DT: $n=$ 26/86, 30.2\% [ $n=26 / 63,41 \cdot 3 \%$ evaluable patients]). More FCT than DT recipients were also 'very satisfied' or 'satisfied' with their medication overall at SOT (FCT: $n=$ $67 / 87,77.0 \%[n=67 / 70,95.7 \%$ evaluable patients $]$ and DT: $n=40 / 86,46.5 \%[n=40 / 70,57.1 \%$ evaluable patients]) and EOT (FCT: $n=56 / 87,64 \cdot 4 \%$ [ $n=56 / 60,93 \cdot 3 \%$ evaluable patients] and DT: $n=28 / 86,32.6 \%[n=28 / 63$, $44.4 \%$ evaluable patients]).

\section{Item results from the $\mathrm{mSICT}$ concerns domain}

The concerns domain consisted of three questions related to: worries about not swallowing enough medication, medication limiting usual activities, and feeling upset about side effects of medication. Overall, patients in the FCT arm reported consistent mean concern scores from SOT (13.8) to EOT (13.7), whereas overall scores in the DT arm worsened at EOT (11.8) compared to $\operatorname{SOT}(12 \cdot 9$, Fig. 1c) and were generally lower (indicating more concerns). For all questions, there were minimal concerns for both medications at SOT and EOT (Fig. 3a, b and c). There was a general trend for more patients in the FCT than the DT group reporting 'never' or 'rarely' feeling concerned at SOT and EOT.

\section{Item results from the Palatability Questionnaire}

The Palatability Questionnaire consisted of four questions to assess taste, aftertaste, whether the medication was taken (e.g., swallowed or vomited), and how the patient perceived the amount of medication (liquid) to be taken. Overall, patients in the FCT arm reported consistently high mean palatability scores from SOT (10.8) to EOT (10.9), where a score of 11 was equivalent to the best outcome, whereas overall scores in the DT arm were lower (9.0 at SOT and 8.8 at EOT) and more variable at each time point (Fig. 1d).

Most patients in both the FCT and DT arms swallowed all their medication at SOT (FCT: $n=68 / 87,78.2 \%[n=$ 68/70, 97.1\% evaluable patients] and DT: $n=68 / 86$, $79.1 \%[n=68 / 69,98.6 \%$ evaluable patients]) and EOT (FCT: $n=60 / 87,69.0 \% \quad[n=60 / 60,100.0 \%$ evaluable patients $]$ and DT: $n=59 / 86,68.6 \%[n=59 / 63,93.7 \%$ evaluable patients]; Fig. 4a). No patient vomited within $30 \mathrm{~min}$ of swallowing their medication. The majority of FCT and DT recipients confirmed that the amount of liquid they took with their medicine for iron overload on that given day was 'just enough' both at SOT (FCT: $n=$ $63 / 87,72.4 \%[n=63 / 70,90 \cdot 0 \%$ evaluable patients $]$ and DT: $n=53 / 86,61.6 \% \quad[n=53 / 69, \quad 76.8 \% \quad$ evaluable patients]) and EOT (FCT: $n=52 / 87,59.8 \%[n=52 / 60$, $86.7 \%$ evaluable patients] and DT: $n=49 / 86,57.0 \%$ [ $n=$ 49/63, 77.8\% evaluable patients]); however, more DT than FCT recipients reported that it was 'too much' (SOT: $n=15 / 86,17.4 \% \quad[n=15 / 69,21.7 \%$ evaluable patients] versus $n=5 / 87,5 \cdot 8 \%[n=5 / 70,7 \cdot 1 \%$ evaluable patients]; EOT: $n=12 / 86,14.0 \%[n=12 / 63,19.0 \%$ evaluable patients] versus $n=5 / 87,5 \cdot 8 \%$ [ $n=5 / 60,8 \cdot 3 \%$ evaluable patients]; Fig. 4b). Nearly all patients in the FCT group reported no issue with the taste $(100 \%$ at SOT and EOT) or aftertaste (98\% at SOT and 100\% EOT) of their medicine; whereas $\sim 20 \%$ of patients $(\sim 30 \%$ evaluable patients) at SOT and EOT in the DT group reported that their medication had a 'bad' or 'very bad' taste or aftertaste (Fig. 4c and d). 

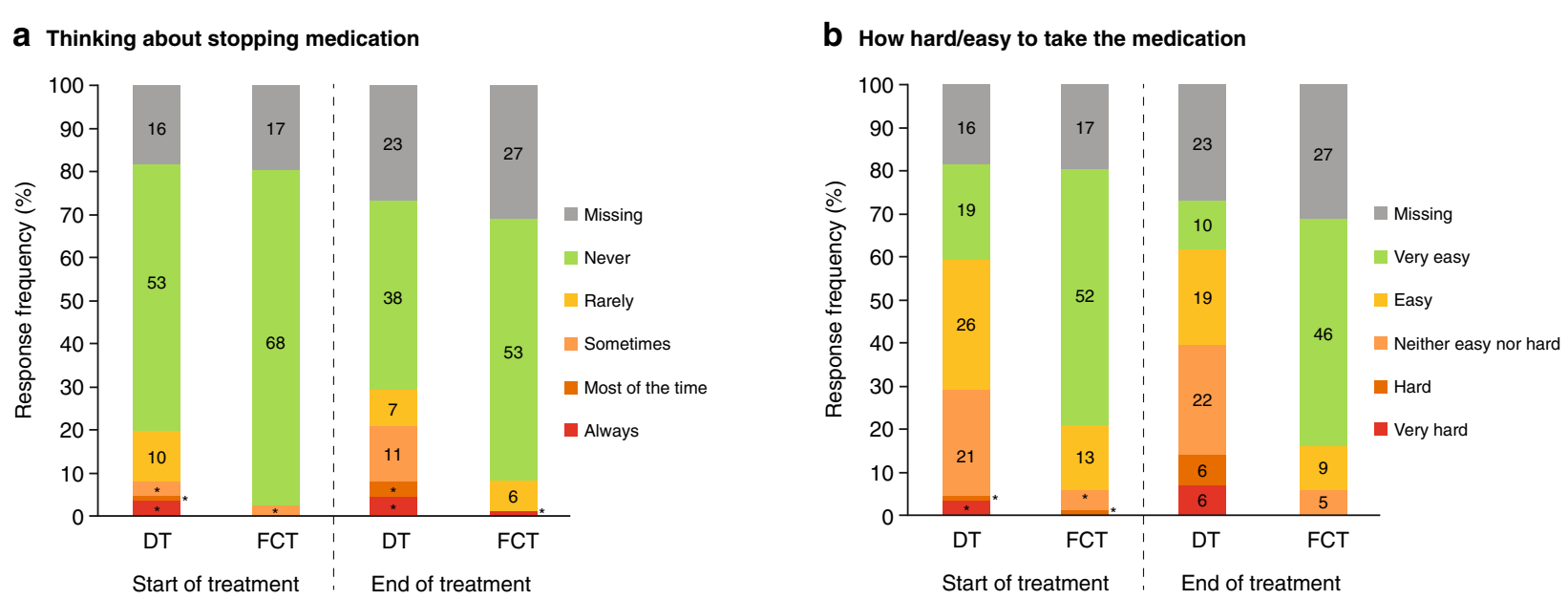

\section{Bothered about time it took to prepare medication}

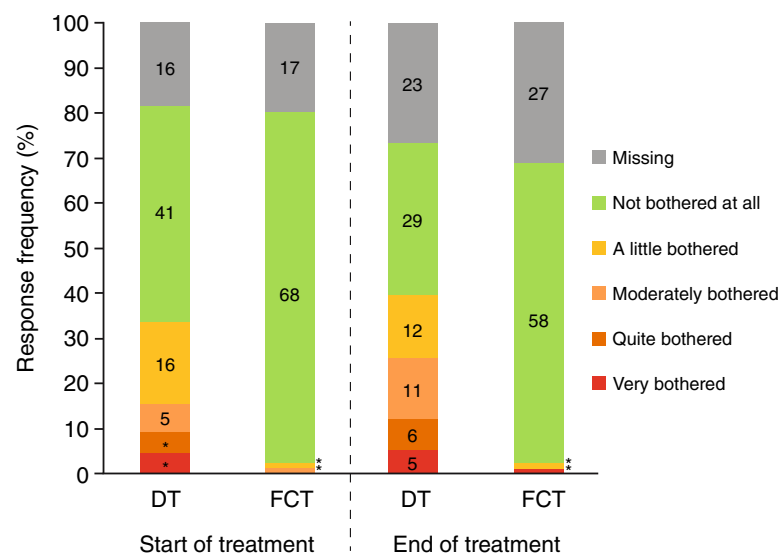

\section{d Bothered about waiting time to eat}

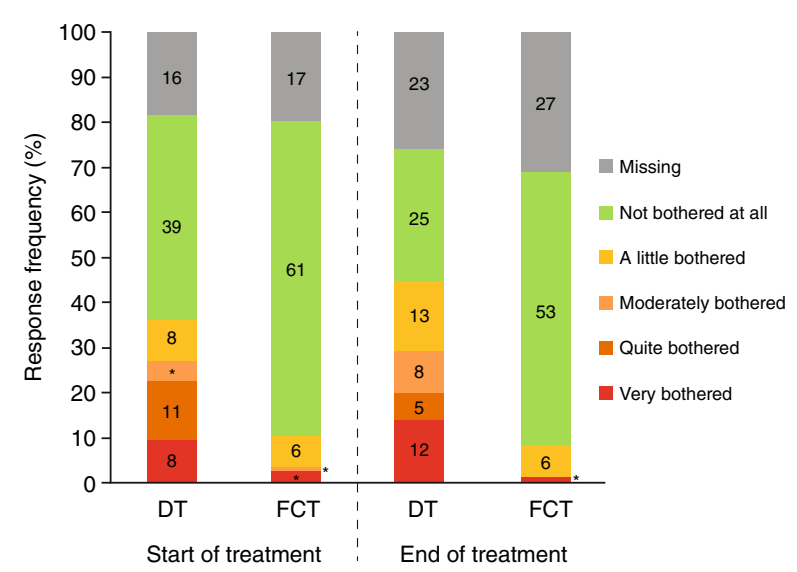

\section{e Following doctor's instructions}

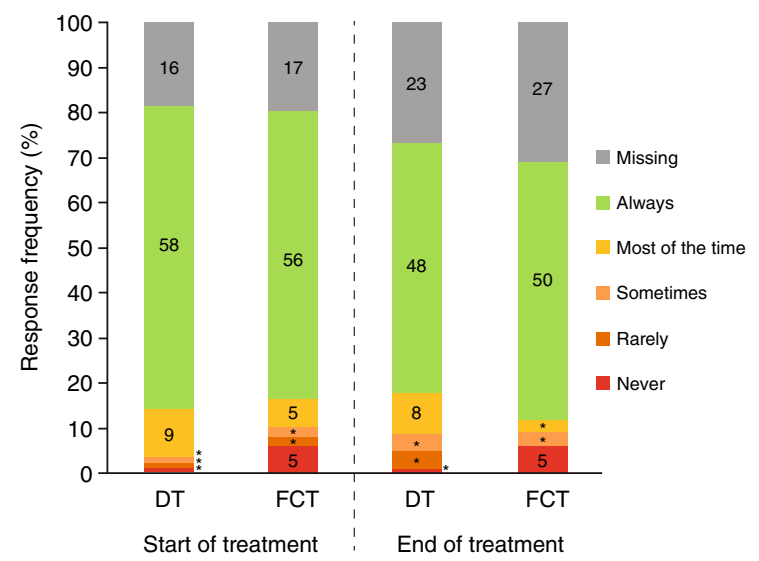

\section{f Trouble remembering to take medication}

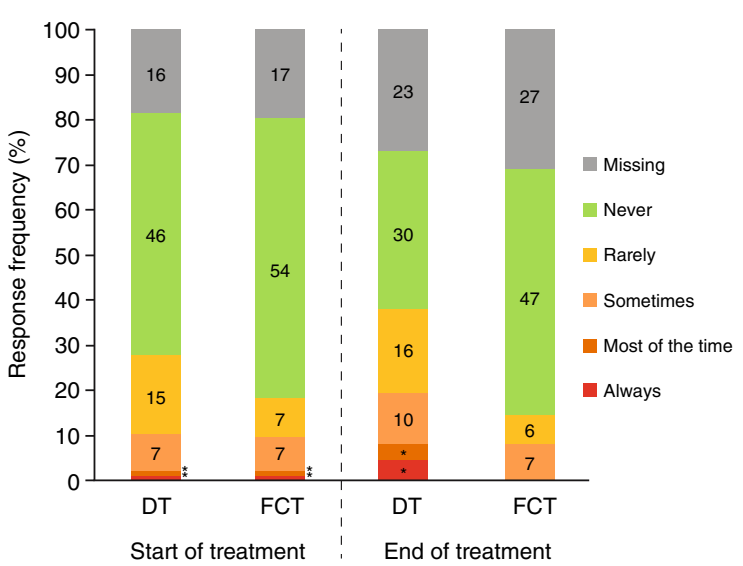

Fig. 2 Response frequencies at week 2 (start of treatment) and end of treatment by treatment arm from the mSICT adherence domains, a Thinking about stopping medication, $\mathbf{b}$ How hard/easy to take the medication, c Time to prepare medication, $\mathbf{d}$ Waiting time to eat, e Following doctor's instructions, and $\mathbf{f}$ Trouble remembering to take medication. Numbers indicate the number of patients in each response category; ${ }^{*} n<5$ patients. DT, dispersible tablet; FCT, film-coated tablet; mSICT, modified Satisfaction with Iron Chelation Therapy 

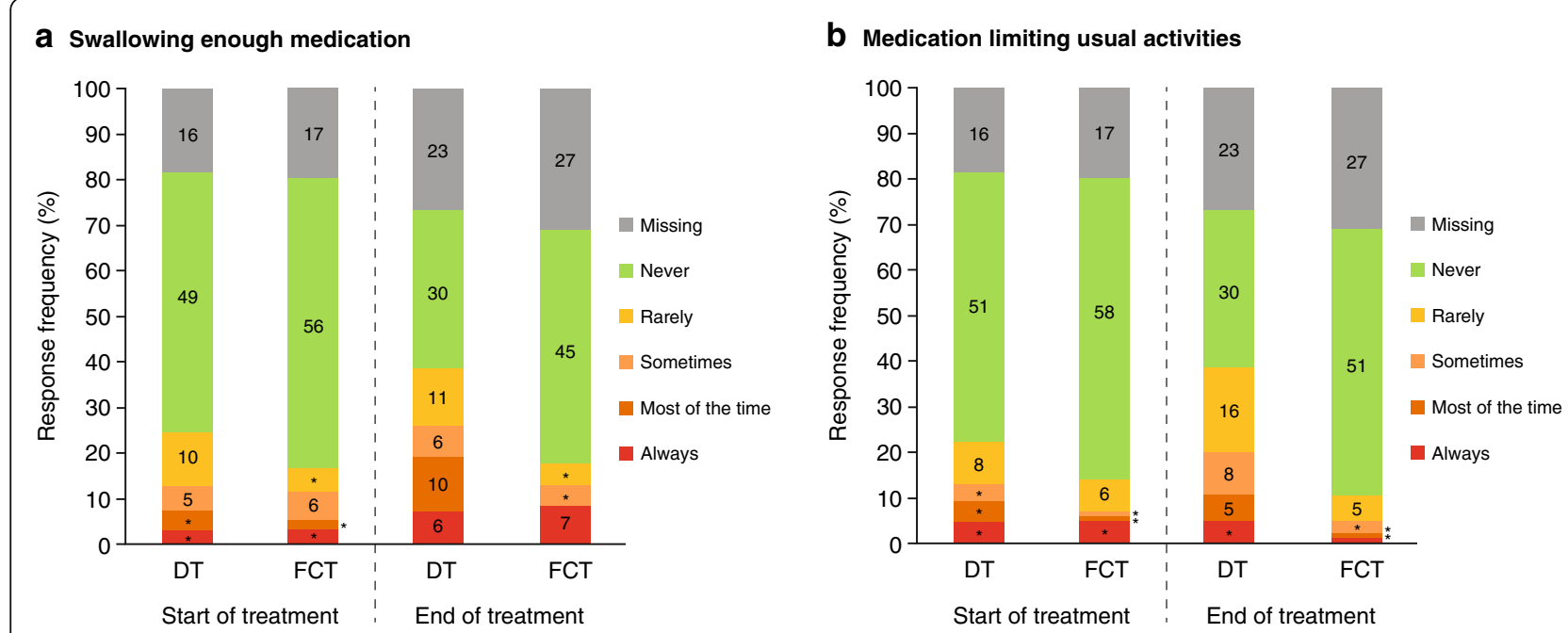

\section{Feeling upset about side effects}

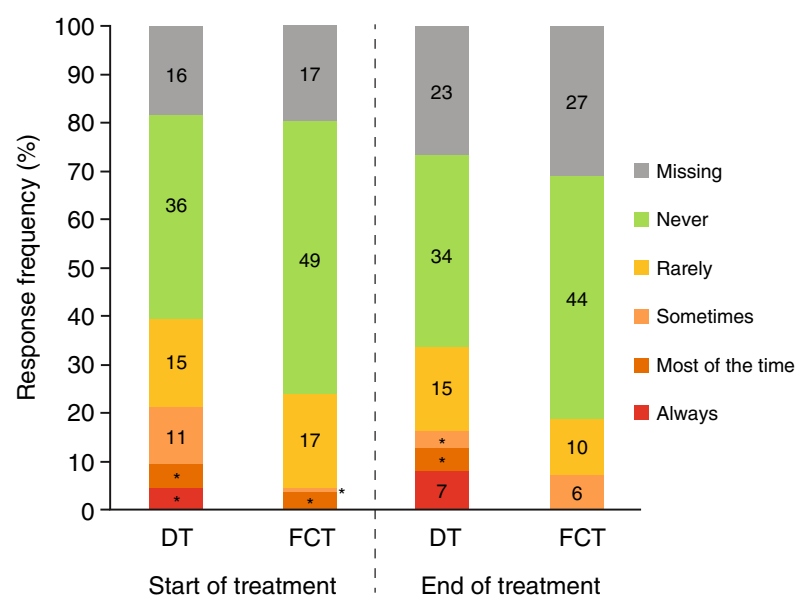

Fig. 3 Response frequencies at week 2 (start of treatment) and end of treatment by treatment arm from the mSICT concerns domains, a Swallowing enough medication, $\mathbf{b}$ Medication limiting usual activities, $\mathbf{c}$ Feeling upset about side effects. Numbers indicate the number of patients in each response category; ${ }^{*} n<5$ patients. DT, dispersible tablet; $F C$, film-coated tablet; mSICT, modified Satisfaction with Iron Chelation Therapy

\section{Overall preference}

Evaluation of the preferred formulation of deferasirox was assessed as part of the mSICT satisfaction domain. In patients who received either the DT or the FCT, there was a preference for the FCT (Fig. 5). At EOT, of patients in the FCT arm, 53/87 (60.9\% [ $n=53 / 60,88.3 \%$ evaluable patients]) reported preference for the FCT, while $3 / 87(3.5 \%[n=3 / 60,5.0 \%$ evaluable patients $])$ preferred a DT. Of patients in the DT arm, $41 / 86(47.7 \%$ [ $n=41 / 63,65.1 \%$ evaluable patients]) reported preference for a FCT, while 16/86 (18.6\% $[n=16 / 63,25.4 \%$ evaluable patients]) preferred the DT.

\section{GI Symptom Diary}

Completion of the GI Symptom Diary also decreased over the course of the study (Table 1). As previously reported [20], the overall GI symptom scores were low for both formulations over the course of the study (less than 3 out of a maximum of 50; Fig. 1e), indicating that patients experienced very little concern associated with GI symptoms. Analysis of summary scores by underlying anemia or age group also did not reveal any notable differences compared to the overall findings (see Additional file 1: Table S2).

\section{Discussion}

It has been well documented that adherence to iron chelation therapy influences overall survival [2, 4, 27]. Although the introduction of once-daily oral chelation with deferasirox DT was an improvement over parenteral DFO, the properties of deferasirox DT were still not ideal. The deferasirox FCT formulation was specifically developed with the intention of improving patient acceptability, to enhance patient satisfaction and therefore adherence to iron chelation therapy. Besides evaluating the safety profile 

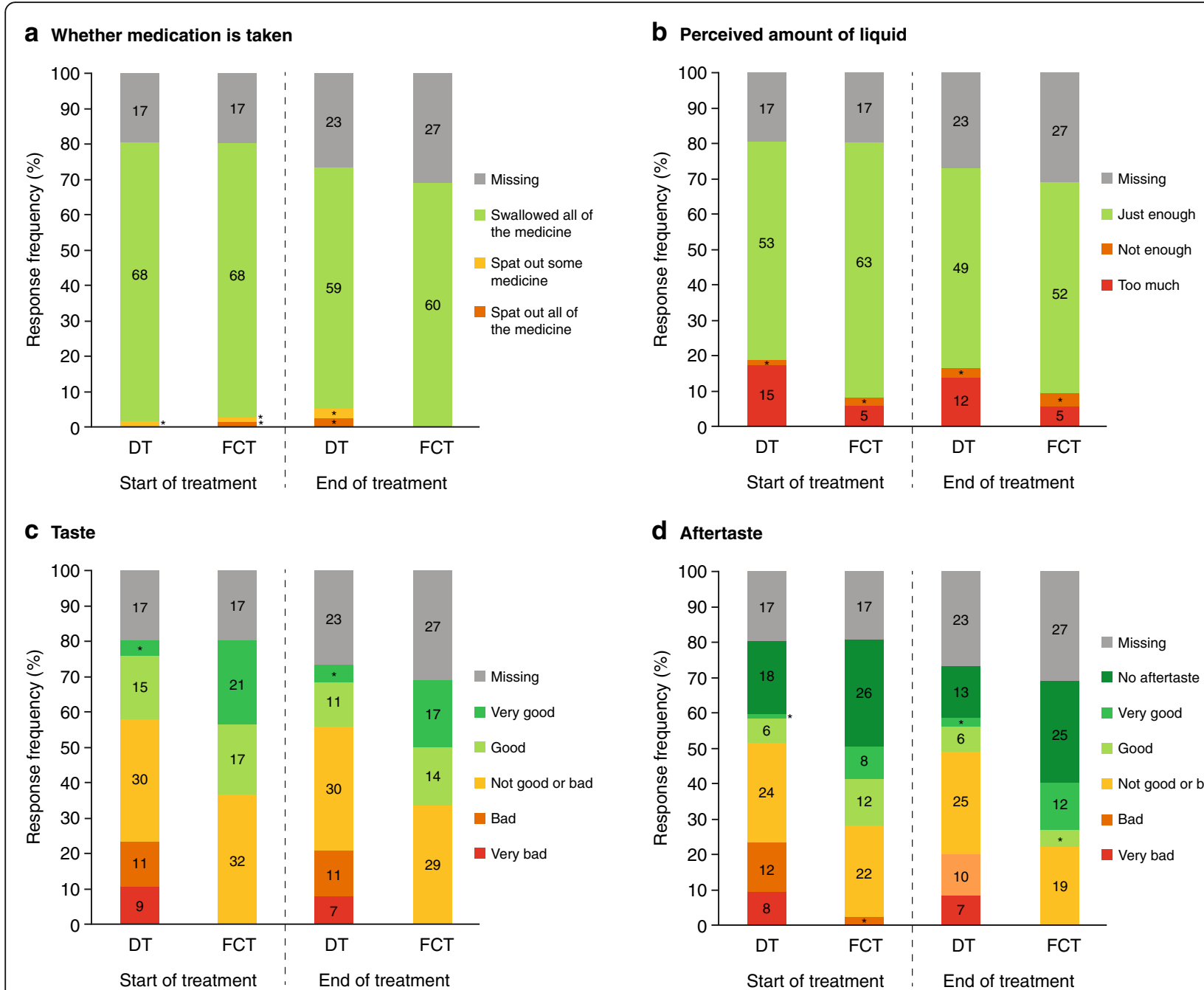

d Aftertaste

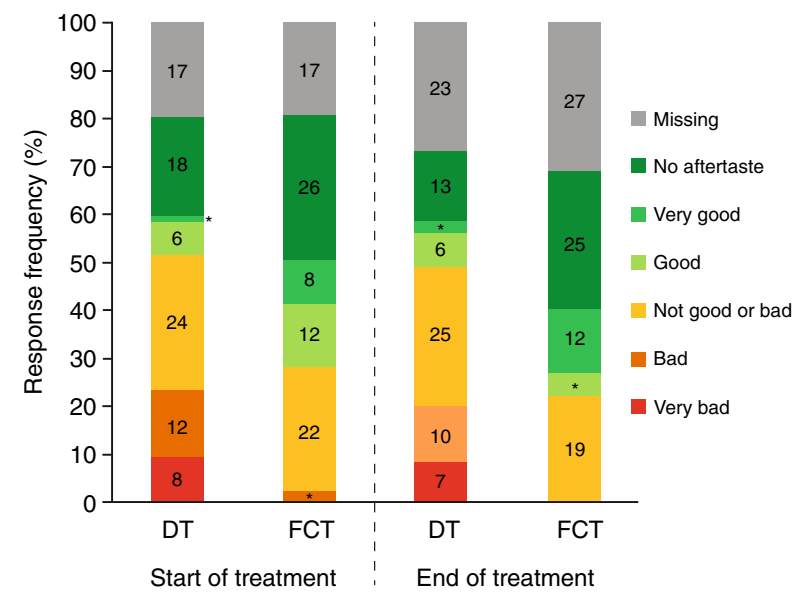

Fig. 4 Response frequencies at week 2 (start of treatment) and end of treatment by treatment arm from the Palatability Questionnaire items, a Whether medication was taken, $\mathbf{b}$ Perceived amount of liquid, c Taste, and $\mathbf{d}$ Aftertaste. Numbers indicate the number of patients in each response category; ${ }^{*} \mathrm{n}<5$ patients; DT, dispersible tablet; $\mathrm{FCT}$, film-coated tablet

and pharmacokinetics of the deferasirox FCT relative to the DT formulation, the ECLIPSE trial also examined detailed PRO data to better understand patient preference for, and satisfaction with, the FCT versus the DT. In general, the PROs demonstrated that patients receiving either the FCT or DT had no major issues regarding adherence, preference, satisfaction, palatability, and GI tolerability with their medication, with patients providing mostly neutral or positive responses to the questionnaires. However, patients receiving FCT and DT showed a clear patient preference for the deferasirox FCT over the DT formulation. Furthermore, these findings were maintained irrespective of underlying anemia or age group, indicating consistent outcomes across patient subgroups. As previously reported, the mean domain scores from the mSICT and Palatability Questionnaires showed adherence, preference, satisfaction, and palatability in favor of the deferasirox FCT [20]. Furthermore, the difference in scores between treatment groups was of a magnitude that implies a corresponding treatment benefit (the difference being $>1$ point $[\mathrm{MID}=1]$ ), indicating a clinically meaningful difference. However, the GI symptom score diaries revealed no clear differences between the two deferasirox formulations, with the majority of patients showing GI disturbances of minimal concern over the 24-week study. This may be attributed to the majority of patients enrolled having experienced prior chelation therapy and having underlying thalassemia, in whom GI disturbances are usually mild-to-moderate [28]. Nevertheless, the analyses presented here show that item results from the mSICT and Palatability Questionnaires provide several specific underlying factors that can be considered as reasons for patients preferring the deferasirox FCT to a degree that may improve their clinical outcomes. 


\section{Preferred formulation of deferasirox}

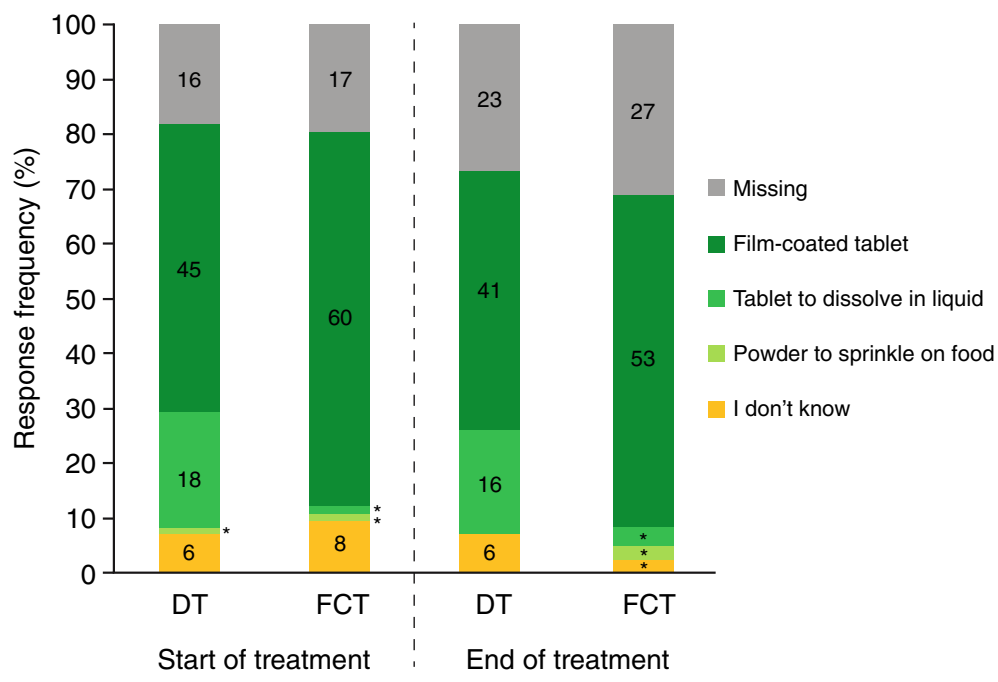

Fig. 5 Response frequencies for the preferred formulation of deferasirox at week 2 (start of treatment) and end of treatment by treatment arm. Numbers indicate the number of patients in each response category; ${ }^{*} \mathrm{n}<5$ patients; DT, dispersible tablet; FCT, film-coated tablet

The adherence domain outcomes from the mSICT showed that all FCT and DT patients generally followed their doctor's instructions when taking their medication, had minimal trouble remembering to take their medication, and rarely thought about stopping. However, by EOT a slightly lower proportion of patients in the DT arm reported 'never or rarely' having trouble remembering to take their medication compared to the FCT arm, as well as 'never' thinking about stopping. The finding that the majority of patients receiving the FCT formation found it 'easy' or 'very easy' to take their medication compared with those in the DT arm, along with not being bothered by the time it took to take their medication or waiting to eat, suggests that the FCT may provide a more convenient mode of administration. The FCT can be taken either on an empty stomach or with a low-fat meal $(<7 \%$ fat content and $\sim 250 \mathrm{cal})$ [19], whereas it is recommended that the DT is taken only on an empty stomach at least $30 \mathrm{~min}$ before the next meal, following dispersion in a glass of water, orange juice or apple juice [18], which takes approximately 3 min to prepare. Previous studies have indicated that the ability to take deferasirox with food, at breakfast in particular, is important to patients [17]. The mode and convenience of administration of the FCT also may have contributed to the majority of patients feeling satisfied with their medication and experiencing fewer concerns, such as worry over swallowing enough medication or impact on their usual activities. Further study is warranted to corroborate these findings.

Patient responses to items on the Palatability Questionnaire revealed similar trends as those observed for the mSICT items, whereby patients receiving the FCT trended towards reporting more positive response. Some patients receiving the DT reported that it tasted 'bad' or 'very bad.' However minimal issues with taste or aftertaste were reported by patients receiving the FCT. The majority of FCT and DT recipients also confirmed that the amount of liquid they took with their medicine was 'just enough'. On the other hand, more DT recipients compared to FCT recipients indicated that the amount of liquid was 'too much', because of the need to disperse their medication in $\sim 200 \mathrm{~mL}$ (for doses $\geq 1 \mathrm{~g}, \sim 100 \mathrm{~mL}$ for doses $<1 \mathrm{~g}$ ). Taking all these factors into account, adherence, satisfaction, concerns, and palatability, there was a clear preference for the FCT over the DT both at week 2 (SOT) and EOT.

This study was limited by the short duration of followup -24 weeks is a relatively short period of time in patients receiving chronic treatment for iron overload that can last a lifetime - however, apparent differences were notable between the two formulations both at 2 weeks (SOT) and at EOT. In addition, not all patients completed the various PRO instruments (attributed to missed patient visits, patients forgetting to bring their PRO devices or oversight in completing the questionnaires during site visits), which may have led to some bias in the findings. However, the completion rate was similar for both formulations and as expected in a PRO study, with a decline in response rate over the course of the study, particularly for the GI questionnaire. This may have been the result of the requirement for daily completion during the study, which some patients may have found burdensome. It should also be noted that the 
completion rates were based on the FAS and were not adjusted to account for decreasing numbers of patients (discontinuations) over the 24-week study. Furthermore, this was a descriptive, exploratory study. Additional long-term experiences from patients in clinical practice treated with the FCT, as well as other clinical trials with longer treatment duration, are required to confirm whether the improved satisfaction, preference, and adherence shown for FCT are maintained over longer time periods. With a short-term safety profile consistent with the known deferasirox DT [20] - well characterized in an extensive clinical trial program - longer follow-up of patients receiving the FCT will also help determine whether the improvements in PROs will translate into better clinical outcomes. In particular, patients switching from the DT to the FCT will require careful monitoring to avoid potential 'over-chelation' with improved adherence, even with the initial FCT dose reduction (e.g., $20 \mathrm{mg} / \mathrm{kg} /$ day DT equivalent to $14 \mathrm{mg} / \mathrm{kg} /$ day FCT; conversion factor 1.43).

\section{Conclusions}

In conclusion, for all patients regardless of age group or underlying disease, these findings from the detailed analyses of responses to various validated PRO instruments could suggest a preference in favor of the once-daily deferasirox FCT formulation. The FCT, which contains the same active ingredient as the DT but with excipients removed, and can be taken orally once daily with or without a light meal, may therefore be less burdensome to patients than the DT formulation. Together with a safety profile consistent with the known deferasirox DT formulation, as previously reported [20], the deferasirox FCT may improve patient experience with iron chelation therapy resulting in greater adherence. It is anticipated that better adherence will translate into a reduction in the frequency and severity of iron overload-related complications.

\section{Additional file}

Additional file 1: Table S1. Summary of domain scores for the mSICT and palatability PRO instruments, by week, treatment and underlying anemia or age group. Table S2. Summary of domain scores for the Gl Symptom Diary, by week, treatment and underlying anemia or age group. (DOCX $45 \mathrm{~kb})$

\section{Abbreviations}

Cl: Confidence interval; DFO: Deferoxamine; DFX: Deferasirox; DT: Dispersible tablet; EOT: End of treatment; FAS: Full analysis set; FCT: Film-coated tablet; Gl: Gastrointestinal; MDS: Myelodysplastic syndromes; MID: Minimally important difference; mSICT: Modified satisfaction with iron chelation therapy; PRO: Patient-reported outcome; SOT: Start of treatment; TDT: Transfusion-dependent thalassemia; ULN: Upper limit of normal

\section{Acknowledgements}

We thank Rebecca Helson, PhD, of Mudskipper Business Ltd. for medical writing assistance. Financial support for medical writing assistance was provided by Novartis Pharmaceuticals Corporation.

\section{Funding}

This study was funded by Novartis Pharma AG.

\section{Availability of data and materials}

The datasets supporting the conclusions of this article are available on clinicaltrials.gov (https://clinicaltrials.gov/ct2/show/NCT02125877) and/or are included within the article (and supplementary information).

\section{Data sharing}

Novartis is committed to sharing with qualified external researchers access to patient-level data and supporting clinical documents from eligible studies. These requests are reviewed and approved by an independent review panel on the basis of scientific merit. All data provided are anonymised to respect the privacy of patients who have participated in the trial, in line with applicable laws and regulations. This trial data availability is in accordance with the criteria and process described on www.clinicalstudydatarequest.com.

\section{Authors' contributions}

$A T T, R O, S P, A K o, G B R, A K a, A-S G$ and JBP served as investigators in this trial, enrolling patients. $\mathrm{VH}$ and $\mathrm{RMH}$ contributed to the analysis, interpretation and reporting of the trial data. AZ served as the trial statistician. All authors contributed to data interpretation, reviewed and provided their comments on this manuscript, and approved the final version.

\section{Authors' information}

Not applicable.

\section{Ethics approval and consent to participate}

The study was conducted in accordance with Good Clinical Practice guidelines and the Declaration of Helsinki and was approved by independent ethics committees at participating sites. Patients (or parents/guardians) provided written, informed consent prior to enrollment.

\section{Consent for publication}

Not applicable.

\section{Competing interests}

ATT reports receiving research funding and honoraria from Novartis and research funding from Celgene Corporations; RO reports receiving honoraria from Novartis and Apopharma, and for being part of the Italian advisory board for BlueBird Bio; SP reports receiving research funding and honoraria from Novartis and research funding from Acceleron; AKo reports receiving honoraria from Novartis, Amgen, and Janssen, and consultancy for Gilead, Roche, and Celgene; AKa reports receiving research funding by Novartis and participating in advisory boards and educational forums sponsored from Novartis, Apopharma, and Celgene; JBP reports consultancy, receiving research grant funding and honoraria from Novartis Pharmaceuticals, consultancy and honoraria from Shire, and consultancy for Celgene. JBP is supported by the NIHR University College London Hospitals Biomedical Research Centre; VH, AZ, and RMH were full-time employees of Novartis at the time of these analyses; GBR and A-SG declares that they have no competing interests.

\section{Publisher's Note}

Springer Nature remains neutral with regard to jurisdictional claims in published maps and institutional affiliations.

\footnotetext{
Author details

${ }^{1}$ Department of Internal Medicine, Director - Fellowship and Residents Research Program, Faculty of Medicine American University of Beirut Medical Center, Beirut, Lebanon. 'Ospedale Pediatrico Microcitemico 'A.Cao', A.O. 'G. Brotzu', Cagliari, Italy. 'Università della Campania "L. Vanvitelli,", Caserta, Italy. ${ }^{4}$ Hematology Division, Department of Internal Medicine, University of Patras Medical School, Patras, Greece. ${ }^{5}$ U.O.C. Ematolog. Con Talassemia, A.O. Civico-Di Cristina-Benfratelli, Palermo, Italy. ${ }^{6}$ First Department of Pediatrics, University of Athens, Athens, Greece. ${ }^{7}$ Department of Medicine, Hospital Pulau Pinang, Georgetown, Penang, Malaysia. ${ }^{8}$ Novartis Pharmaceuticals
} 
Corporation, East Hanover, NJ, USA. ${ }^{9}$ Novartis Pharma AG, Basel, Switzerland. ${ }^{10}$ University College London, London, UK. ${ }^{11}$ Emory School of Medicine, Atlanta, USA.

Received: 9 October 2017 Accepted: 31 October 2018

Published online: 19 November 2018

\section{References}

1. Payne KA, Rofail D, Baladi JF, Viala M, Abetz L, Desrosiers MP, Lordan N, Ishak K, Proskorovsky I. Iron chelation therapy: clinical effectiveness, economic burden and quality of life in patients with iron overload. Adv Ther. 2008;25:725-42.

2. Delea TE, Edelsberg J, Sofrygin O, Thomas SK, Baladi J-F, Phatak PD, Coates TD. Consequences and costs of noncompliance with iron chelation therapy in patients with transfusion-dependent thalassemia: a literature review. Transfusion. 2007:47:1919-29.

3. Escudero-Vilaplana V, Garcia-Gonzalez X, Osorio-Prendes S, Romero-Jimenez RM, Sanjurjo-Saez M. Impact of medication adherence on the effectiveness of deferasirox for the treatment of transfusional iron overload in myelodysplastic syndrome. J Clin Pharm Ther. 2016;41:59-63.

4. Gabutti V, Piga A. Results of long-term iron-chelating therapy. Acta Haematol. 1996;95:26-36.

5. Cappellini MD, Cohen A, Piga A, Bejaoui M, Perrotta S, Agaoglu L, Aydinok Y, Kattamis A, Kilinc Y, Porter J, Capra M, Galanello R, Fattoum S, Drelichman G, Magnano C, Verissimo M, Athanassiou-Metaxa M, Giardina B, KourakliSymeonidis A, Janka-Schaub G, Coates T, Vermylen C, Olivieri N, Thuret I, Opitz H, Ressayre-Djaffer C, Marks P, Alberti D. A phase 3 study of deferasirox (ICL670), a once-daily oral iron chelator, in patients with betathalassemia. Blood. 2006;107:3455-62

6. Cappellini MD, Porter JB, El-Beshlawy A, Li C-K, Seymour JF, Elalfy M, Gattermann N, Giraudier S, Lee J-W, Chan LL, Lin K-H, Rose C, Taher A, Thein SL, Viprakasit V, Habr D, Domokos G, Roubert B, Kattamis A. On behalf of the EPIC study investigators. Tailoring iron chelation by iron intake and serum ferritin: the prospective multicenter EPIC study of deferasirox in 1744 patients with various transfusion-dependent anemias. Haematologica. 2010;95:557-66.

7. Cappellini MD, Bejaoui M, Agaoglu L, Canatan D, Capra M, Cohen A, Drelichman G, Economou M, Fattoum S, Kattamis A, Kilinc Y, Perrotta S, Piga A, Porter JB, Griffel L, Dong V, Clark J, Aydinok Y. Iron chelation with deferasirox in adult and pediatric patients with thalassemia major: efficacy and safety during 5 years' follow-up. Blood. 2011;118:884-93.

8. Galanello R, Piga A, Forni GL, Bertrand Y, Foschini ML, Bordone E, Leoni G, Lavagetto A, Zappu A, Longo F, Maseruka H, Hewson N, Sechaud R, Belleli $\mathrm{R}$, Alberti D. Phase II clinical evaluation of deferasirox, a once-daily oral chelating agent, in pediatric patients with $\beta$-thalassemia major. Haematologica. 2006;91:1343-51.

9. Pennell DJ, Porter JB, Cappellini MD, El-Beshlawy A, Chan LL, Aydinok Y, Elalfy MS, Sutcharitchan P, Li CK, Ibrahim H, Viprakasit V, Kattamis A, Smith G, Habr D, Domokos G, Roubert B, Taher A. Efficacy of deferasirox in reducing and preventing cardiac iron overload in $\beta$-thalassemia. Blood. 2010;115:2364-71.

10. Piga A, Galanello R, Forni GL, Cappellini MD, Origa R, Zappu A, Donato G, Bordone E, Lavagetto A, Zanaboni L, Sechaud R, Hewson N, Ford JM, Opitz $\mathrm{H}$, Alberti D. Randomized phase II trial of deferasirox (Exjade ${ }^{\oplus}$, ICL670), a once-daily, orally-administered iron chelator, in comparison to deferoxamine in thalassemia patients with transfusional iron overload. Haematologica. 2006:91:873-80

11. Porter J, Galanello R, Saglio G, Neufeld EJ, Vichinsky E, Cappellini MD, Olivieri N, Piga A, Cunningham MJ, Soulières D, Gattermann N, Tchernia G, Maertens J, Giardina P, Kwiatkowski J, Quarta G, Jeng M, Forni GL, Stadler M, Cario H, Debusscher L, Della Porta M, Cazzola M, Greenberg P, Alimena G, Rabault B, Gathmann I, Ford JM, Alberti D, Rose C. Relative response of patients with myelodysplastic syndromes and other transfusion-dependent anaemias to deferasirox (ICL670): a 1-yr prospective study. Eur J Haematol. 2008;80:168-76.

12. Vichinsky E, Onyekwere $O$, Porter J, Swerdlow P, Eckman J, Lane P, Files B, Hassell K, Kelly P, Wilson F, Bernaudin F, Forni GL, Okpala I, Ressayre-Djaffer C, Alberti D, Holland J, Marks P, Fung E, Fischer R, Mueller BU, Coates T. A randomized comparison of deferasirox versus deferoxamine for the treatment of transfusional iron overload in sickle cell disease. Br J Haematol. 2007;136:501-8
13. Vichinsky E, Bernaudin F, Forni GL, Gardner R, Hassell K, Heeney MM, Inusa B, Kutlar A, Lane P, Mathias L, Porter J, Tebbi C, Wilson F, Griffel L, Deng W, Giannone V, Coates T. Long-term safety and efficacy of deferasirox (Exjade ${ }^{\circledast}$ ) for up to 5 years in transfusional iron-overloaded patients with sickle cell disease. Br J Haematol. 2011;154:387-97.

14. Cappellini MD, Bejaoui M, Agaoglu L, Porter J, Coates T, Jeng M, Lai ME, Mangiagli A, Strauss G, Girot R, Watman N, Ferster A, Loggetto S, Abish S, Cario H, Zoumbos N, Vichinsky E, Opitz H, Ressayre-Djaffer C, Abetz L, Rofail D, Baladi J-F. Prospective evaluation of patient-reported outcomes during treatment with deferasirox or deferoxamine for iron overload in patients with $\beta$-thalassemia. Clin Ther. 2007;29:909-17.

15. Vichinsky E, Pakbaz Z, Onyekwere $O$, Porter J, Swerdlow $P$, Coates T, Lane $P$, Files B, Mueller BU, Coic L, Forni GL, Fischer R, Marks P, Rofail D, Abetz L, Baladi J-F. Patient-reported outcomes of deferasirox (Exjade ${ }^{\oplus}$, ICL670) versus deferoxamine in sickle cell disease patients with transfusional hemosiderosis: substudy of a randomized open-label phase II trial. Acta Haematol. 2008;119:133-41.

16. Osborne RH, De Abreu LR, Dalton A, Houltram J, Dowton D, Joshua DE, Lindeman R, Ho PJ. Quality of life related to oral versus subcutaneous iron chelation: a time trade-off study. Value Health. 2007;10:451-6.

17. Goldberg SL, Giardina PJ, Chirnomas D, Esposito J, Paley C, Vichinsky E. The palatability and tolerability of deferasirox taken with different beverages or foods. Pediatr Blood Cancer. 2013;60:1507-12.

18. Novartis Pharmaceuticals. EXJADE ${ }^{\oplus}$ (deferasirox) US prescribing information. 2015. Available at: http://www.pharma.us.novartis.com/product/pi/pdf/exjade.pdf.

19. Novartis Pharmaceuticals. JADENU ${ }^{\otimes}$ (deferasirox) US prescribing information. 2015. Available at: www.pharma.us.novartis.com/product/pi/pdf/jadenu.pdf.

20. Taher AT, Origa R, Perrotta S, Kourakli A, Ruffo GB, Kattamis A, Goh AS, Cortoos A, Huang V, Weill M, Merino HR, Porter JB. New film-coated tablet formulation of deferasirox is well tolerated in patients with thalassemia or lower-risk MDS: results of the randomized, phase II ECLIPSE study. Am J Hematol. 2017:92:420-8

21. Velez-Nandayapa L, Holder G, Horowitz J, Singal R, Cortoos A, Eisinger J. Post-marketing safety of two different formulations of deferasirox, filmcoated tablet (FCT) and dispersible tablet (DT), a comparative assessment. Drug Safety. 2016;39:989.

22. Lasch K, Cote I, Roma T, Srivastava B, Horodniceanu EG, Dhatt H, Carter JA, Bal V. Modification of patient reported outcomes measures of compliance, gastrointestinal symptoms, palatability and treatment satisfaction for patients needing iron chelation therapy. Value Health. 2014;17:A574.

23. Huang W, Banderas B, Sen R. Psychometric evaluation of clinical outcomes assessments in a Phase II trial. Vienna: International Society for Pharmacoeconomics and Outcomes Research (ISPOR-EU) 19th Annual European Congress; 2016. p. PCN210.

24. Norman GR, Sloan JA, Wyrwich KW. Interpretation of changes in healthrelated quality of life: the remarkable universality of half a standard deviation. Med Care. 2003;41:582-92.

25. Copay AG, Subach BR, Glassman SD, Polly DW Jr, Schuler TC. Understanding the minimum clinically important difference: a review of concepts and methods. Spine J. 2007;7:541-6.

26. Schunemann HJ, Guyatt GH. Commentary--goodbye M(C)ID! Hello MID, where do you come from? Health Serv Res. 2005;40:593-7.

27. Delea TE, Sofrygin O, Thomas SK, Baladi J-F, Phatak PD, Coates TD. Cost effectiveness of once-daily oral chelation therapy with deferasirox versus infusional deferoxamine in transfusion-dependent thalassaemia patients. US healthcare system perspective. Pharmacoeconomics. 2007; 25:329-42.

28. Vichinsky E. Clinical application of deferasirox: practical patient management. Am J Hematol. 2008;83:398-402. 